\title{
Fabrication and room temperature operation of semiconductor nano-ring lasers using a general applicable membrane transfer method
}

Cite as: Appl. Phys. Lett. 110, 171105 (2017); https://doi.org/10.1063/1.4982621

Submitted: 19 February 2017 . Accepted: 08 April 2017 . Published Online: 26 April 2017

Fan Fan, Yueyang Yu, Seyed Ebrahim Hashemi Amiri, David Quandt (D), Dieter Bimberg, and C. Z. Ning
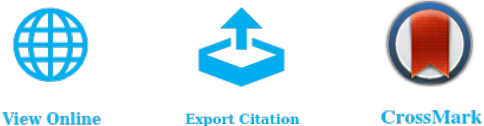

\section{ARTICLES YOU MAY BE INTERESTED IN}

Continuous-wave operation and $10-\mathrm{Gb} / \mathrm{s}$ direct modulation of InAsP/InP sub-wavelength nanowire laser on silicon photonic crystal

APL Photonics 2, 046106 (2017); https://doi.org/10.1063/1.4977927

High temperature limit of semiconductor nanowire lasers

Applied Physics Letters 110, 173103 (2017); https://doi.org/10.1063/1.4982629

Second-order distributed-feedback surface plasmon resonator for single-mode fiber endfacet biosensing

Applied Physics Letters 110, 171107 (2017); https://doi.org/10.1063/1.4982625

\section{Lock-in Amplifiers up to $600 \mathrm{MHz}$}
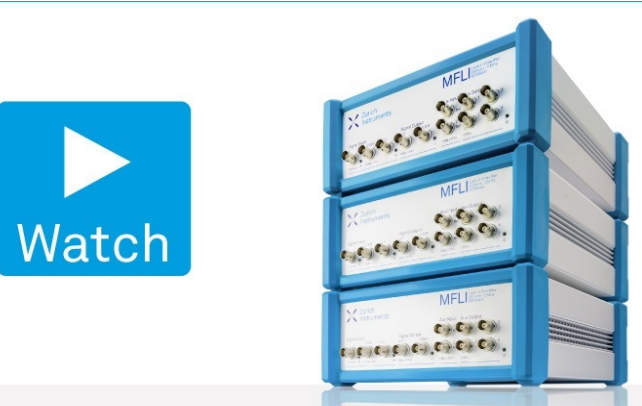


\title{
Fabrication and room temperature operation of semiconductor nano-ring lasers using a general applicable membrane transfer method
}

\author{
Fan Fan, ${ }^{1,2}$ Yueyang Yu, ${ }^{1}$ Seyed Ebrahim Hashemi Amiri, ${ }^{1}$ David Quandt, ${ }^{3}$ \\ Dieter Bimberg, ${ }^{3,4}$ and C. Z. Ning ${ }^{1,2, a)}$ \\ ${ }^{1}$ School of Electrical, Computer, and Energy Engineering, Arizona State University, Tempe, Arizona 85287, \\ USA \\ ${ }^{2}$ Department of Electronic Engineering, Tsinghua University, Beijing 100084, China \\ ${ }^{3}$ Institut für Festkörperphysik, Technische Universität Berlin, Berlin 10623, Germany \\ ${ }^{4}$ King Abdulaziz University, Jeddah 21589, Saudi Arabia
}

(Received 19 February 2017; accepted 8 April 2017; published online 26 April 2017)

\begin{abstract}
Semiconductor nanolasers are potentially important for many applications. Their design and fabrication are still in the early stage of research and face many challenges. In this paper, we demonstrate a generally applicable membrane transfer method to release and transfer a strain-balanced InGaAs quantum-well nanomembrane of $260 \mathrm{~nm}$ in thickness onto various substrates with a high yield. As an initial device demonstration, nano-ring lasers of $1.5 \mu \mathrm{m}$ in outer diameter and $500 \mathrm{~nm}$ in radial thickness are fabricated on $\mathrm{MgF}_{2}$ substrates. Room temperature single mode operation is achieved under optical pumping with a cavity volume of only $0.43 \lambda_{0}^{3}$ ( $\lambda_{0}$ in vacuum). Our nano-membrane based approach represents an advantageous alternative to other design and fabrication approaches and could lead to integration of nanolasers on silicon substrates or with metallic cavity. Published by AIP Publishing.

[http://dx.doi.org/10.1063/1.4982621]
\end{abstract}

Semiconductor nanolasers have attracted a great deal of interest $^{1-3}$ in the last decade or so. Tremendous progress has been made in various innovative designs, physics understanding, fabrication, and device demonstration, driven mostly by promising application in small foot-print on-chip interconnects in future large-scale nanophotonic integrated systems. Early nanolasers were demonstrated in the form of semiconductor nanowires ${ }^{1,3}$ or photonic crystal lasers. ${ }^{4-6}$ Both types of lasers represent significant reduction of sizes compared to more traditional semiconductor lasers but are still large in certain dimensions or overall sizes. To reduce the size of lasers even further, it was obvious that pure semiconductor or dielectric cavity structures are insufficient. Metal-coated semiconductor nanowires were thus investigated as a mechanism for further size reduction, ${ }^{7,8}$ where plasmonic effects ${ }^{7}$ allowed much dramatic size reduction. Many different variations of metallic cavity nanolaser designs have been proposed and studied in the meantime. ${ }^{7-21}$ These activities have resulted in unprecedented size reduction of semiconductor lasers and culminated in the first demonstration of room temperature (RT), continuous wave operation of metal-semiconductor nanolasers under electrical injection with a size smaller than the wavelength (in vacuum). ${ }^{21}$

Despite initial success in metal-semiconductor based nanolasers over the last few decades, significant issues remain. ${ }^{22}$ First, many characteristics of nanolasers need to be improved such as lifetimes, threshold, and efficiency. Second, reproducible and high-yield fabrication still poses significant challenges. One of the critical issues that affect the fabrication complexity and performance is the use of dielectric insulating materials such as $\mathrm{Si}_{3} \mathrm{~N}_{4}$ or $\mathrm{SiO}_{2}$ to separate the metal shell from the semiconductor core. Such dielectric layers hinder

\footnotetext{
${ }^{\text {a) }}$ Author to whom correspondence should be addressed. Electronic mail: cning@asu.edu
}

thermal dissipation and cause mechanical breakdown at high pumping levels. Thus, search for alternative device designs and fabrication routes remains necessary.

Another parallel development is silicon ( $\mathrm{Si}$ ) photonics, where Si-based lasers are desired for compatibility with complementary metal-oxide-semiconductors (CMOSs). Various early attempts have been made to achieve heterogeneous integration on a $\mathrm{Si}$ platform. ${ }^{23-31}$ The membrane transfer approach is considered one of the promising techniques and has attracted great deals of interest ever since the first demonstration in $2006 .^{32}$ Significant progress has been made recently in the membrane transfer method ${ }^{33-38}$ for fabrication of III-V devices on Si. Even though lasers have been reported using such membrane transfer techniques, ${ }^{35-38}$ challenges remain with the release of the membrane to different substrates. The success of the transfer method depends on the adhesion energy between the semiconductor membrane to be transferred and the elastomeric stamp used and that between the membrane and the receiving substrate. Often, the yield of release depends on the peeling speed. High peeling speed is employed for releasing the membrane from the donor substrate to the stamp, while low peeling speed is employed for releasing the membrane from the stamp onto the receiving substrate. Thus, the successful release and transfer of the membrane often depends on the surface properties of the membrane and the receiving substrates and is sensitively dependent on the peeling speed of the stamps, making it difficult to release and transfer the membrane layer in a controllable manner, to a variety of diverse substrates, with a high yield.

In addition to providing a means for fabricating III-V devices on $\mathrm{Si}$, the membrane transfer approach also offers considerable benefits for nanolaser fabrication to remedy some of the deficiencies mentioned above. The membrane transfer approach allows much thinner layers to be used as a device structure with a better index contrast to a carefully 
selected new receiving substrate than on the original growth substrate. The removal of the original thick substrate represents a significant reduction of the overall device volume. The fabricated devices without the original substrate can have much better thermal dissipation if transferred to a metal-film or other good thermal conductor. As described earlier, heat dissipation is one of the serious issues for nanolasers with oxide or dielectric isolating layers. Finally, the membrane transfer approach permits much more integration flexibility such as on various substrates and potentially coupling with Si-, plasmonic, or other dielectric waveguides.

In this paper, we demonstrate an alternative membrane transfer approach using an intermediate layer between the membrane and the polydimethylsiloxane (PDMS) stamps, as demonstrated recently for the fabrication of Graphene based devices. ${ }^{39}$ This method does not require the control of the peeling speed and the associated calibration and greatly simplifies the membrane transfer procedure. Using this approach, we demonstrated the general applicability and control in transferring a nano-membrane onto $\mathrm{MgF}_{2}$ substrates with a high yield. The subsequent fabrication of laser devices based on the transferred membranes demonstrates the overall success of this approach. Arrays of nano-ring lasers of $1.5 \mu \mathrm{m}$ in diameter with a radial width of only $500 \mathrm{~nm}$ and thickness of $260 \mathrm{~nm}$ are fabricated. The lasers can operate up to room temperature under optical pumping. While the membrane transfer technique has been used so far to make lasers of tens of microns in lateral dimensions with large thickness, our nanomembrane lasers are likely the smallest lasers fabricated using the membrane technique.

The details of our wafer structure are shown in Table I. The wafer was designed to have a membrane of $254.5 \mathrm{~nm}$ in thickness to be transferred. The semiconductor membrane is epitaxially grown on a GaAs substrate with a $100 \mathrm{~nm}$ AlAs sacrificial layer for selective etching and has a p-i-n structure, consisting of $40 \mathrm{~nm}$-thick doped InGaP layers on each

TABLE I. Wafer design for the membrane structure.

\begin{tabular}{lccccc}
\hline \hline $\begin{array}{l}\text { Layer } \\
\text { No. }\end{array}$ & Material & $\begin{array}{c}\text { Thickness } \\
(\mathrm{nm})\end{array}$ & $\begin{array}{c}\text { Refractive } \\
\text { index }\end{array}$ & $\begin{array}{c}\text { Bandgap } \\
(\mathrm{eV})\end{array}$ & Description \\
\hline 0 & $\mathrm{GaAs}^{41-43}$ & 8 & 3.52 & 1.423 & Cap \\
1 & $\mathrm{Al}_{0.95} \mathrm{Ga}_{0.05} \mathrm{As}$ & 10 & 2.98 & 2.609 & Cap \\
2 & $\mathrm{In}_{0.49} \mathrm{Ga}_{0.51} \mathrm{P}(\mathrm{n})$ & 40 & 3.23 & 1.758 & n-contact \\
3 & $\mathrm{GaAs}_{0.883} \mathrm{P}_{0.117}$ & 20.4 & 3.47 & 1.564 & Barrier \\
4 & $\mathrm{In}_{0.145} \mathrm{Ga}_{0.855} \mathrm{As}$ & 7.9 & 3.56 & 1.217 & QW \\
5 & $\mathrm{GaAs}_{0.883} \mathrm{P}_{0.117}$ & 14.3 & 3.47 & 1.564 & Barrier \\
6 & $\mathrm{In}_{0.145} \mathrm{Ga}_{0.855} \mathrm{As}$ & 7.9 & 3.56 & 1.217 & QW \\
7 & $\mathrm{GaAs}_{0.883} \mathrm{P}_{0.117}$ & 14.3 & 3.47 & 1.564 & Barrier \\
8 & $\mathrm{In}_{0.145} \mathrm{Ga}_{0.855} \mathrm{As}$ & 7.9 & 3.56 & 1.217 & QW \\
9 & $\mathrm{GaAs}_{0.883} \mathrm{P}_{0.117}$ & 14.3 & 3.47 & 1.564 & Barrier \\
10 & $\mathrm{In}_{0.145} \mathrm{Ga}_{0.855} \mathrm{As}$ & 7.9 & 3.56 & 1.217 & QW \\
11 & $\mathrm{GaAs}_{0.883} \mathrm{P}_{0.117}$ & 14.3 & 3.47 & 1.564 & Barrier \\
12 & $\mathrm{In}_{0.145} \mathrm{Ga}_{0.855} \mathrm{As}$ & 7.9 & 3.56 & 1.217 & QW \\
13 & $\mathrm{GaAs}_{0.883} \mathrm{P}_{0.117}$ & 20.4 & 3.47 & 1.564 & Barrier \\
14 & $\mathrm{In}_{0.49} \mathrm{Ga}_{0.51} \mathrm{P}(\mathrm{p})$ & 40 & 3.23 & 1.758 & p-contact \\
15 & $\mathrm{Al}_{0.95} \mathrm{Ga}_{0.05} \mathrm{As}$ & 10 & 2.98 & 2.609 & Cap \\
16 & $\mathrm{GaAs}_{16}$ & 8 & 3.52 & 1.423 & Cap \\
17 & $\mathrm{AlAs}$ & 100 & 2.95 & $\ldots$ & Sacrificial \\
18 & & & & & layer \\
& $\mathrm{GaAs}$ & $\ldots$ & 3.52 & $\ldots$ & Substrate \\
\hline \hline & & & & &
\end{tabular}

side and an intrinsic gain core region. The core region contains five quantum wells of $\mathrm{In}_{0.145} \mathrm{Ga}_{0.885} \mathrm{As}$ (thickness $7.9 \mathrm{~nm}$ ) with $\mathrm{GaAs}_{0.883} \mathrm{P}_{0.117}$ (thickness $14.3 \mathrm{~nm}$ ) as barriers. The carefully strain compensated design, through fine tuning of thickness and composition between the quantum well and barrier layers, and the overall structure symmetry with respect to the middle quantum well (layer no. 8) are critical in producing a flat membrane upon release from the original growth substrate without bending or curling.

To prepare the wafer sample for cross-sectional scanning electron microscopy (SEM), the wafer was first laterally etched so that the AlAs sacrificial layer can be easily identified. A Au layer was deposited on top of the wafer vertical to the cross-sectional plane to increase the conductivity of the sample for better SEM image quality. Due to the composition difference between different epitaxial layers and the resulting image contrast, the multi-quantum-well- (MQW) structure of the membrane was visually observable under the secondary electron mode of the SEM, as shown in Fig. 1(a). The actual thickness of the overall membrane structure was measured to be $260 \mathrm{~nm}$ and the actual thickness of the AlAs sacrificial layer was measured to be $120 \mathrm{~nm}$. To measure the photoluminescence (PL) spectrum, a passively mode-locked Ti:sapphire laser $(790 \mathrm{~nm}, 80 \mathrm{MHz}, 150 \mathrm{fs})$ was used for excitation. As shown in Fig. 1(b), the PL has a peak wavelength at $965 \mathrm{~nm}$ with a full width at half maximum (FWHM) of $17 \mathrm{~nm}$. Our theoretical calculation of the confined states for electrons and holes in the MQW structure (as specified in Table I) resulted in a transition energy of $1.275 \mathrm{eV}$ or wavelength of $972 \mathrm{~nm}$, which is in great agreement with the measured PL peak, well within the experimental margin of errors. The design strategies of the similar MQW structure can be found in Ref. 44 .

The membrane release and transfer process including device fabrication is schematically shown in Fig. 2. After cleaning, the wafer was patterned into $250 \mu \mathrm{m} \times 250 \mu \mathrm{m}$ squares by a AZ3312 photoresist (PR) (Fig. 2(b)). This is followed by a 2-min inductively coupled plasma (ICP) etching step with $2.5 \mathrm{sccm} \mathrm{Cl} \mathrm{Cl}_{2}$ and $35 \mathrm{sccm} \mathrm{BCl}$ at 4 mTorr in a etch tool to create $40 \mu \mathrm{m}$-wide trenches on the wafer (Fig. 2(c)). We intentionally left the photoresist in portions of the etched trenches before immersing the sample into a $2 \% \mathrm{HF}$ solution for $20 \mathrm{~min}$ to selectively under-etch the AlAs layer. The residual PR served to anchor the MQW membrane square mesas to the GaAs substrate preventing them from free floating (Fig. 2(d)). After the release of the membrane through selective under-etching, the pickup-transfer is typically carried out by using a PDMS layer. ${ }^{32-36}$ However, releasing the
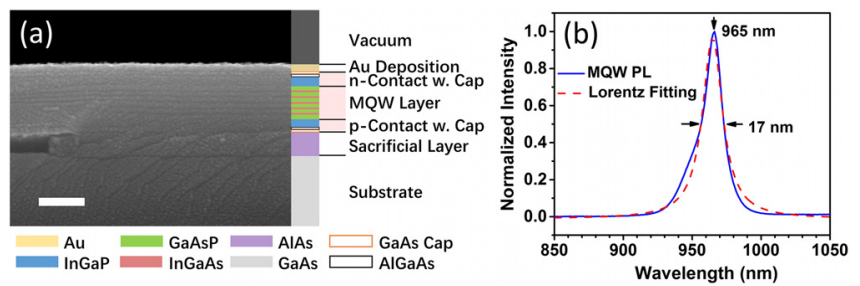

FIG. 1. Characterization of the wafer structure. (a) Cross-sectional SEM image of the wafer structure with composition and layer illustration. The total thickness of the membrane to be transferred is indicated between the Au layer and the sacrificial layer. Scale bar: $200 \mathrm{~nm}$. (b) Photoluminescence spectrum of the MQW structure with Lorentz fitting. 
(a)

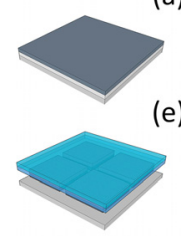

(e)

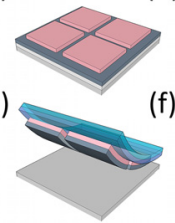

(b)

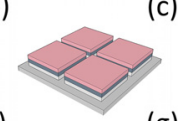

(i)

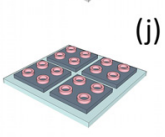

(j)

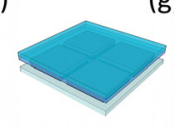

(g)

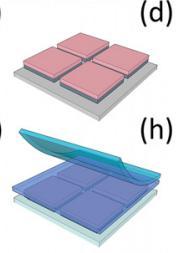

(d)

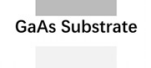

(h)

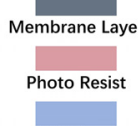

(k)

(I)
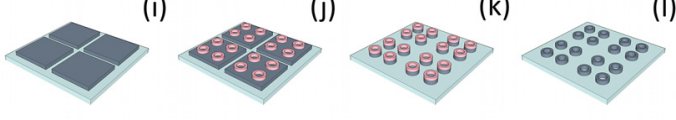

PPC Film

PDMS Film

$\mathrm{MgF}_{2}$ Substrate

FIG. 2. Membrane release-transfer and nano-ring laser fabrication process: (a) Starting wafer as shown in Table I; (b) patterning with photoresist AZ3312 to define $250 \mu \mathrm{m} \times 250 \mu \mathrm{m}$ squares over the entire wafer; (c) ICP etching down through the AlAs sacrificial layer to produce trenches for under-etching; (d) $2 \%$ HF wet under-etching of the AlAs sacrificial layer; (e) and (f) membrane pick-up by a PDMS-PPC bilayer structure; (g) membrane transfer onto a new receiving substrate $\left(\mathrm{MgF}_{2}\right.$ in this work); (h) PDMS removal; (i) PPC and PR dissolution by acetone; (j) laser structure patterning; (k) ICP etching; and (l) PR cleaning to finalize the laser devices.

membrane from PDMS stamps to the new receiving substrate turns out to be often problematic because of strong adhesion between PDMS and the semiconductor membranes. The standard methods hitherto rely on the control of the peeling speed at which the PDMS is peel-removed from the membranes, leaving the membranes onto the receiving substrate. Due to the different surface adhesion conditions between the semiconductor membranes and the receiving substrates, extensive calibration of peeling speed through numerous experiments is required. This becomes prohibitively expensive for III-V semiconductor heterostructure membranes. To have a more generally applicable and systematic transfer procedure that works for different types of semiconductor membranes and receiving substrates, we adopted an approach that has been demonstrated recently in graphene device fabrication, ${ }^{37}$ in which a polypropylene carbonate (PPC) film is used as an intermediate layer between PDMS and the semiconductor membrane. The PPC film was first spin coated on a PDMS stamp. The semiconductor membrane was next picked up by the PPC-PDMS bilayer from the original growth substrate with the PPC layer facing down in direct contact with the semiconductor membrane (Figs. 2(e) and 2(f)). After releasing the membrane from the growth substrate, the PPCPDMS bilayer was heated up to $110^{\circ} \mathrm{C}$ together with the membranes to further soften the PPC layer. The bilayer stamp with the membrane stack was then attached onto a receiving substrate. After cooling down to below the glass transition temperature, the PPC layer became hardened and detached from the soft PDMS layer. Therefore, the PDMS layer could then be easily removed from the stack (Fig. 2(h)), leaving the membrane and the PPC layer on top of the receiving substrate. Thereafter, both PPC and PR residues were dissolved by acetone and completely cleaned by an $\mathrm{O}_{2}$ plasma (Fig. 2(i)). Since the releasing step in the entire transfer process only deals with the adhesion between PPC and PDMS, this procedure is applicable in general for different types of substrates and membrane combinations. We had tested the membrane transfer on various receiving substrates, including $\mathrm{Si}$ substrates, Au-deposited substrates, $\mathrm{SiO}_{2}$ substrates, and $\mathrm{MgF}_{2}$ substrates with transfer yield $>80 \%$. In this work,

$\mathrm{MgF}_{2}$ substrates were used as the receiving substrate for the fabrication of nanolasers due to their low refractive index, which gives better optical modal confinement for the semiconductor membrane and better thermal conductivity than $\mathrm{SiO}_{2}$. Ring shaped arrays were then patterned onto the transferred membranes (Fig. 2(j)) via photolithography and an identical dry etching step was applied to finalize the laser patterns (Fig. 2(k)), followed by a final PR clean step (Fig. 2(1)).

Fig. 3(a) shows an SEM image of a transferred $250 \mu \mathrm{m}$ $\times 250 \mu \mathrm{m}$ nano-membrane square. Due to the strain compensation of our quantum-well membrane design, no curving or bending was observed after the sacrificial under etching, leading to the successful transfer of entire membrane squares. This transfer approach has been applied to fabricate various membrane lasers of ring and disk shapes, with outer diameters of rings in the range of 1.5-1.7 $\mu \mathrm{m}$ and disk diameters in the range of $2-5 \mu \mathrm{m}$. Nano-ring laser devices of $1.5 \mu \mathrm{m}$ in outer diameter are shown in Fig. 3(b). The inner diameters of the $1.5 \mu \mathrm{m}$ nano-ring lasers are $500 \mathrm{~nm}$ on average and the radius thicknesses are around $500 \mathrm{~nm}$. For the membrane of $260 \mathrm{~nm}$ in thickness, the cavity volumes of the $1.5 \mu \mathrm{m}$ nano-ring lasers are $0.43 \lambda_{0}{ }^{3}$ ( $\lambda_{0}$ : wavelength in vacuum) or $16.9 \lambda^{3}$ with $\lambda$ being the wavelength in the material.

The nano-ring lasers were characterized at room temperature under optical pumping of a $349 \mathrm{~nm}$ pulsed laser (Spectra Physics, $1 \mathrm{kHz}, 5 \mathrm{~ns}$ ) at an angle of $45^{\circ}$ from the sample normal with the beam size around $20 \mu \mathrm{m}$. PL spectra evolution under increased pumping strengths is presented in Fig. 4(a), showing a clear single mode operation due to the compact cavity volume of the nano-ring lasers. At lowest pumping, very weak broadband spontaneous emission was observed, slightly above the noise background. With the increase in the pumping levels from 25.3 to $36.6 \mathrm{~nJ}$, a sharp peak at $980 \mathrm{~nm}$ appeared and the intensity of the peak increased super-linearly. At high pumping levels above $45 \mathrm{~nJ}$, the intensity of the sharp peak was at least 20 times stronger than the broadband emission background and the linewidth of the peak decreased down to $1.2 \mathrm{~nm}$, as measured by a spectrometer with a $600 \mathrm{~g} / \mathrm{mm}$ grating. The linewidth was limited by the large slit size at the spectrometer entrance due to the collection at the normal direction (see more discussions about Fig. 5). The lasing behavior was further confirmed by the light-in-light-out (LILO) curve in Fig. 4(b). Clear threshold behavior and typical S-shaped curve covering the three regimes of operation can be easily observed in the linear-scale plot and double-log-scale plot, respectively. (a)

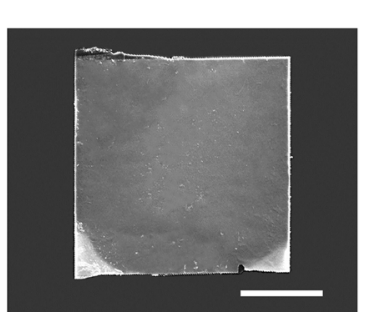

(b)

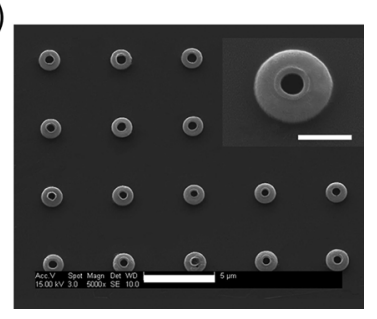

FIG. 3. SEM image of a released membrane and the final nano-ring laser arrays: (a) SEM image of a released membrane of a square shape with a side length of $250 \mu \mathrm{m}$ before nano-ring patterning; scale bar: $100 \mu \mathrm{m}$; (b) SEM image of the fabricated nano-ring laser array; scale bar: $5 \mu \mathrm{m}$. Inset: zoomedin image of a nano-ring laser with an outer diameter of $1.5 \mu \mathrm{m}$, an inner diameter of $500 \mathrm{~nm}$, and a radial thickness of $\sim 500 \mathrm{~nm}$; scale bar: $1 \mu \mathrm{m}$. 

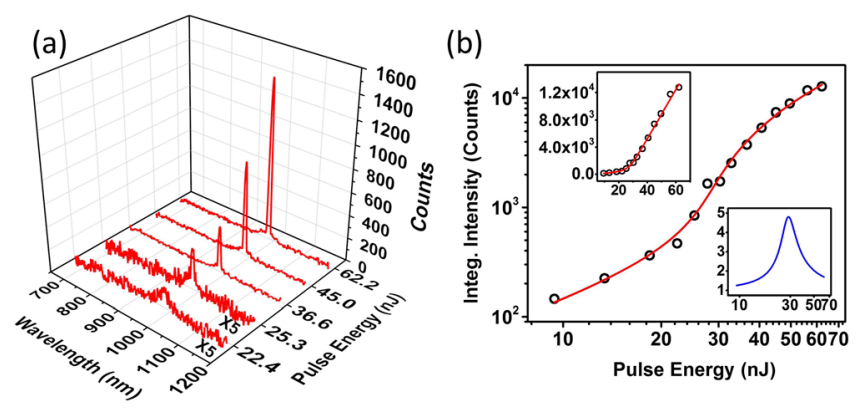

FIG. 4. Lasing characterization of the nano-ring laser devices shown in Fig. 3(b). (a) Spectra evolution of the nano-ring laser with the increase in pumping; (b) experimental data of LILO relation (black circles) on a double logarithmic scale with lasing rate equation fitting (red solid line). Upper left inset: LILO relation on a linear scale. Lower right inset: slope of LILO on a double logarithm scale where the peak (maximum of the slope) indicates the threshold.

The experimental data agree well with a theoretical fitting (solid line in Fig. 4(b)) to the solution of the single mode rate equations. The slopes in double-log scale plot (right bottom inset of Fig. 4(b)) identifies the threshold of the laser at the maximum slope of $29 \mathrm{~nJ}$, equivalent to an average power density of $9 \mathrm{~W} / \mathrm{cm}^{2}$, corresponding to an estimated absorbed power density of $4 \mathrm{~W} / \mathrm{cm}^{2}$.

To further corroborate the experimental results and to gain more understanding, we performed numerical simulation of the nanoring laser using the finite-difference timedomain (FDTD) method with the device parameters as presented above. The results are presented in Fig. 5. A mode with a resonance wavelength of $979.6 \mathrm{~nm}$ was found in our simulation, in close agreement with the measured result. The mode has a predominant polarization of the $z$-component, vertical to the plane of the membrane, and much weaker components in the other directions. The polarization and the mode patterns determine the far-field pattern, which is predominantly in the direction of the membrane plane (see Fig. $5(f)$ ). Such a pattern is ideal for coupling of the ring laser
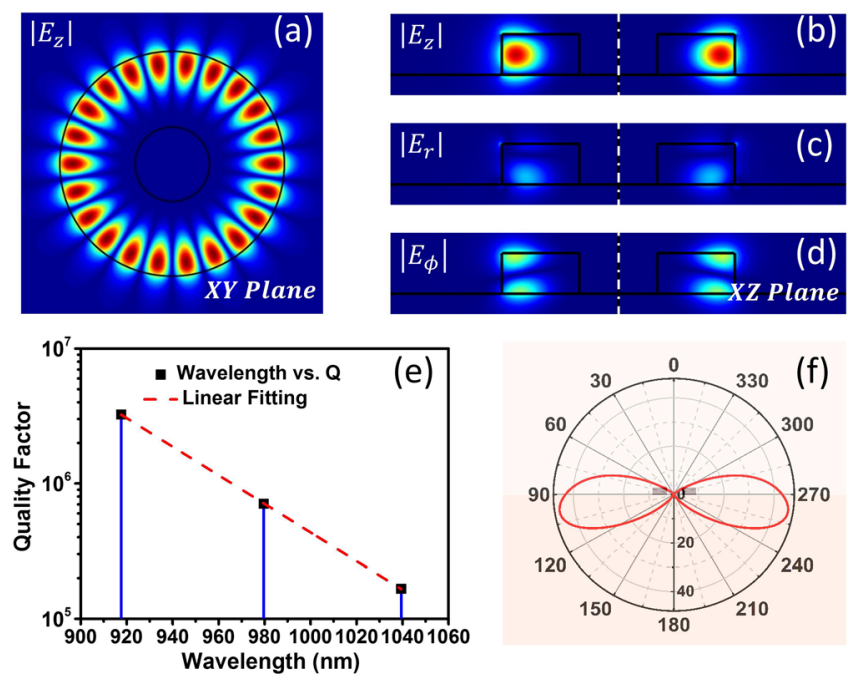

FIG. 5. Simulation results of the laser as fabricated. (a)-(d) Mode pattern of (a) Ez component in the membrane plane (XY plane) and (b)-(d) all three electric components in the cross-sectional plane (XZ plane) vertical to the membrane plane with a resonant wavelength of $979.6 \mathrm{~nm}$ calculated by the FDTD method; (e) the relation of wavelength versus Q of modes near $980 \mathrm{~nm}$; (f) the far-field distribution of relative electric-field intensity in the $\mathrm{XZ}$ plane. into an in-plane tangential waveguide, typical for such coupling. But the far-field pattern is poorly suited for detection in the normal direction. Due to such poor collection geometry, only a small portion of the emission signal was detected from the lasing mode and mostly was the scattered light. This is why we had to use a large entrance slit to the spectrometer, leading to a system-limited linewidth of $\sim 1.2 \mathrm{~nm}$ in Fig. 4(a). The simulated quality factor (Q) of the lasing mode is as large as $7.1 \times 10^{5}$, indicating potentially a very high Q laser using such a small membrane cavity. However, the fabrication imperfection (such as non-uniform thickness of the ring and the non-ideal circular shape seen in the SEM in Fig. 3(b)) has significantly degraded the cavity Q. A more refined and optimized fabrication could improve the device performance, in light of the high theoretical Q value.

In conclusion, we have demonstrated a generally applicable and more systematic approach for semiconductor nanomembrane transfer from the original growth substrates to various receiving substrates. The procedure is a robust one with an overall yield higher than $80 \%$. The method has produced a transfer of the nano-membrane with the thickness as thin as $260 \mathrm{~nm}$. As an initial device demonstration, we have fabricated semiconductor membrane lasers of nano-ring shape with the outer diameter of $1.5 \mu \mathrm{m}$ and the radial thickness of $500 \mathrm{~nm}$, capable of operating at room temperature. The total volume of the nano-ring lasers is only $0.43 \lambda_{0}{ }^{3}$ ( $\lambda_{0}$ in vacuum) on average. The method can be applicable to a wide range of substrates, especially including metallic substrates and $\mathrm{Si}$. Such an approach will allow a wide range of device fabrication and integration, providing a general avenue towards metallic-cavity nanolasers using membrane transfer and Sibased nanolaser. As we mentioned, one of the problems with our current use of dielectric insulating layers in the metallic nanolaser fabrication ${ }^{21}$ is the thermal mismatch and associated mechanical stability. Our thin-membrane laser has already built-in wide gap layers to serve as electronic insulating layers. The thickness of such layers could be further optimized for good electronic insulation and for minimizing metal loss. We believe that the removal of dielectric layers such as $\mathrm{SiO}_{2}$ or $\mathrm{SiN}$ from nanolaser design and the use of such single-step epitaxial membrane will lead to better device stability, performance, and lifetime. All of these potential advantages remain to be demonstrated in the future.

This work was partly supported by the Army Research Office (Grant No. W911NF-13-1-0278), by the 985 Key University Project of China at Tsinghua University, and by SFB 787 of DFG. We thank Xuetao Gan and Kevin Hilger for technical discussions on the transfer approach and device fabrication.

${ }^{1}$ M. H. Huang, S. Mao, H. Feick, H. Yan, Y. Wu, H. Kind, E. Weber, R. Russo, and P. Yang, "Room-temperature ultraviolet nanowire nanolasers," Science 292, 1897 (2001).

${ }^{2}$ C. Z. Ning, "Semiconductor nanolasers," Phys. Status Solidi B 247, 774 (2010).

${ }^{3}$ C. Z. Ning, "Semiconductor nanowire lasers," in Advances in Semiconductor Lasers, Semiconductors and Semimetals, edited by J. J. Coleman, A. C. Bryce, and C. Jagadish (Academic Press, Elsevier, Burlington, 2012), Vol. 86, pp. 455-486. 
${ }^{4}$ O. Painter, R. K. Lee, A. Scherer, A. Yariv, J. D. O’Brien, P. D. Dapkus, and I. Kim, "Two-dimensional photonic band-gap defect mode laser," Science 284, 1819-1821 (1999).

${ }^{5}$ S. Noda, "Photonic crystal lasers-Ultimate nanolasers and broad area coherent lasers," J. Opt. Soc. Am. B 27, B1-B8 (2010).

${ }^{6}$ H. G. Park, S. H. Kim, S. H. Kwon, Y. G. Ju, J. K. Yang, J. H. Baek, S. B. Kim, and Y. H. Lee, "Electrically driven single-cell photonic crystal laser," Science 305, 1444-1447 (2004).

${ }^{7}$ A. V. Maslov and C. Z. Ning, "Size reduction of a semiconductor nanowire laser using metal coating," SPIE Proc. 6468, 64680I (2007).

${ }^{8}$ M. T. Hill, Y.-S. Oei, B. Smalbrugge, Y. Zhu, T. de Vries, P. J. van Veldhoven, F. W. M. van Otten, T. J. Eijkemans, J. P. Turkiewicz, H. de Waardt, E. J. Geluk, S.-H. Kwon, Y.-H. Lee, R. Nötzel, and M. K. Smit, "Lasing in metalliccoated nanocavities," Nat. Photonics 1(10), 589-594 (2007).

${ }^{9}$ M. T. Hill, M. Marell, E. S. P. Leong, B. Smalbrugge, Y. Zhu, M. Sun, P. J. van Veldhoven, E. J. Geluk, F. Karouta, Y. S. Oei, R. Nötzel, C. Z. Ning, and M. K. Smit, Opt. Express 17, 11107 (2009).

${ }^{10}$ D. J. Bergman and M. I. Stockman, "Surface plasmon amplification by stimulated emission of radiation: Quantum generation of coherent surface plasmons in nanosystems," Phys. Rev. Lett. 90, 027402 (2003).

${ }^{11}$ R. F. Oulton, V. J. Sorger, T. Zentgraf, R.-M. Ma, C. Gladden, L. Dai, G. Bartal, and X. Zhang, "Plasmon lasers at deep subwavelength scale," Nature 461, 629-632 (2009).

${ }^{12}$ M. A. Noginov, G. Zhu, A. M. Belgrave, R. Bakker, V. M. Shalaev, E. E. Narimanov, S. Stout, E. Herz, T. Suteewong, and U. Wiesner, "Demonstration of a spaser-based nanolaser," Nature 460, 1110-1113 (2009).

${ }^{13}$ R. Perahia, T. P. Mayer Alegre, A. H. Safavi-Naeini, and O. Painter, "Surface-plasmon mode hybridization in subwavelength microdisk lasers," Appl. Phys. Lett. 95, 201114 (2009).

${ }^{14}$ M. P. Nezhad, A. Simic, O. Bondarenko, B. Slutsky, A. Mizrahi, L. Feng, V. Lomakin, and Y. Fainman, "Room-temperature subwavelength metallo-dielectric lasers," Nat. Photonics 4, 395-399 (2010).

${ }^{15}$ S.-H. Kwon, J.-H. Kang, C. Seassal, S.-K. Kim, P. Regreny, Y.-H. Lee, C. M. Lieber, and H.-G. Park, "Subwavelength plasmonic lasing from a semiconductor nanodisk with silver nanopan cavity," Nano Lett. 10, 3679-3683 (2010).

${ }^{16} \mathrm{~K}$. Yu, A. Lakhani, and M. C. Wu, "Subwavelength metal-optic semiconductor nanopatch lasers," Opt. Express 18, 8790-8799 (2010).

${ }^{17}$ K. Ding, Z. Liu, L. Yin, H. Wang, R. Liu, M. T. Hill, M. J. H. Marell, P. J. van Veldhoven, R. Nötzel, and C. Z. Ning, "Electrical injection, continuous wave operation of subwavelength-metallic cavity lasers at $260 \mathrm{~K}$," Appl. Phys. Lett. 98, 231108 (2011).

${ }^{18}$ K. Ding, Z. C. Liu, L. J. Yin, M. T. Hill, M. J. H. Marell, P. J. van Veldhoven, R. Nöetzel, and C. Z. Ning, "Room-temperature continuous wave lasing in deep-subwavelength metallic cavities under electrical injection," Phys. Rev. B 85, 041301(R) (2012).

${ }^{19}$ C.-Y. Lu, S.-L. Chuang, and D. Bimberg, "Metal cavity surface-emitting nanolasers," IEEE J. Quantum Electron. 48, 114 (2013).

${ }^{20}$ Y.-J. Lu, J. Kim, H.-Y. Chen, C. Wu, N. Dabidian, C. E. Sanders, C.-Y. Wang, M.-Y. Lu, B.-H. Li, X. Qiu, W.-H. Chang, L.-J. Chen, G. Shvets, C.-K. Shih, and S. Gwo, Science 337, 450-453 (2012).

${ }^{21}$ K. Ding, M. T. Hill, Z. C. Liu, L. J. Yin, P. J. van Veldhoven, and C. Z. Ning, "Record performance of electrical injection sub-wavelength metallic-cavity semiconductor lasers at room temperature," Opt. Express 21, 4728-4733 (2013).

${ }^{22}$ K. Ding and C. Z. Ning, "Fabrication challenges of electrical injection metallic cavity semiconductor nanolasers (invited paper)," Semicond. Sci. Technol. 28, 124002 (2013).

${ }^{23}$ M. E. Groenert, W. L. Christopher, A. J. Pitera, V. Yang, H. Lee, R. J. Ram, and E. A. Fitzgerald, "Monolithic integration of room-temperature cw GaAs/AlGaAs lasers on $\mathrm{Si}$ substrates via relaxed graded GeSi buffer layers," J. Appl. Phys. 93, 362-367 (2003).

${ }^{24}$ S. Wirths, R. Geiger, N. V. D. Driesch, G. Mussler, T. Stoica, S. Mantl, Z. Ikonic, M. Luysberg, S. Chiussi, J. M. Hartmann, H. Sigg, J. Faist, D. Buca, and D. Grutzmacher, "Lasing in direct-bandgap GeSn alloy grown on Si," Nat. Photonics 9, 88-92 (2015).
${ }^{25}$ A. W. Fang, H. Park, O. Cohen, R. Jones, M. J. Paniccia, and J. E. Bowers, "Electrically pumped hybrid AlGaInAs-silicon evanescent laser," Opt. Express 14, 9203-9210 (2006).

${ }^{26}$ J. Van Campenhout, P. Rojo-Romeo, P. Regreny, C. Seassal, D. Van Thourhout, S. Verstuyft, L. D. Cioccio, J. M. Fedeli, C. Lagahe, and R. Baets, "Electrically pumped InP-base microdisk lasers," Opt. Express 15, 6744-6749 (2007).

${ }^{27}$ C. Kopp, S. Bernabe, B. B. Bakir, J. Fedeli, R. Orobtchouk, F. Schrank, H. Porte, L. Zimmermann, and T. Tekin, "Silicon photonic circuits: OnCMOS integration, fiber optical coupling, and packaging," IEEE J. Sel. Top. Quantum Electron. 17, 498-509 (2011).

${ }^{28}$ J. J. Rumpler and C. J. Fonstad, "Continuous-wave electrically pumped $1.55-\mu \mathrm{m}$ edge-emitting platelet ridge laser diodes on silicon," IEEE Photonics Tech. Lett. 21, 827-829 (2009).

${ }^{29}$ D. Inoue, T. Hiratani, K. Fukuda, T. Tomiyasu, T. Amemiya, N. Nishiyama, and S. Arai, "High-modulation efficiency operation of GaInAsP/InP membrane distributed feedback laser on Si substrate," Opt. Express 23(22), 29024 (2015).

${ }^{30}$ D. Inoue, T. Hiratani, Y. Atsuji, T. Tomiyasu, T. Amemiya, N. Nishiyama, and S. Arai, "Monolithic integration of membrane-based butt-jointed built-in DFB lasers and p-i-n photodiodes bonded on Si substrate," IEEE J. Sel. Top. Quantum Electron. 21(6), 1502907 (2015).

${ }^{31}$ T. Hiratani, D. Inoue, T. Tomiyasu, Y. Atsuji, K. Fukuda, T. Amemiya, N. Nishiyama, and S. Arai, "Room-temperature continuous-wave operation of membrane distributed-reflector laser," Appl. Phys. Express 8, 112701 (2015).

${ }^{32}$ M. Meitl, Z. Zhu, V. Kumar, K. J. Lee, X. Feng, Y. Y. Huan, I. Adesida, R. G. Nuzzo, and J. A. Rogers, "Transfer printing by kinetic control of adhesion to an elastomeric stamp," Nat. Mater. 5, 33-38 (2006).

${ }^{33}$ A. Carlson, A. M. Bowen, Y. Huang, R. G. Nuzzo, and J. A. Roger, "Transfer printing techniques for materials assembly and micro/nanodevice fabrication," Adv. Mater. 24, 5284-5318 (2012).

${ }^{34}$ H. Yang, D. Zhao, S. Liu, Y. Liu, J. Seo, Z. Ma, and W. Zhou, "Transfer printed nanomembranes for heterogeneously integrated membrane photonics," Photonics 2, 1081-1100 (2015).

${ }^{35}$ H. Yang, D. Zhao, S. Chuwongin, J. Seo, W. Yang, Y. Shuai, J. Berggren, M. Hammar, Z. Ma, and W. Zhou, "Transfer-printed stacked nanomembrane lasers on silicon," Nat. Photonics 6, 615-620 (2012).

${ }^{36}$ D. Zhao, S. Liu, H. Yang, Z. Ma, C. Reuterskiold-Hedlund, M. Hammer, and W. Zhou, "Printed large-area single-mode photonic crystal bandedge surface-emitting lasers on silicon," Sci. Rep. 6, 18860 (2016).

${ }^{37}$ X. Sheng, C. Robert, S. Wang, G. Pakeltis, B. Corbett, and J. A. Rogers, "Transfer printing of fully formed thin-film microscale GaAs lasers on silicon with a thermally conductive interface material," Laser Photonics Rev. 9(4), L17-L22 (2015).

${ }^{38}$ J. Justice, C. Bower, M. Meitl, M. B. Mooney, M. A. Gubbins, and B. Corbett, "Wafer-scale integration of group III-V lasers on silicon using transfer printing of epitaxial layers," Nat. Photonics 6, 610-614 (2012).

${ }^{39}$ L. Wang, I. Meric, P. Y. Huang, Q. Gao, Y. Gao, H. Tran, T. Taniguchi, K. Watanale, L. M. Campos, D. A. Muller, J. Guo, P. Kim, J. Hone, K. L. Shepard, and C. R. Dean, "One-dimensional electrical contact to a twodimensional material," Science 342, 614-617 (2013).

${ }^{40}$ See http://refractiveindex.info/ for refractive indices of binary compound semiconductor (last accessed March 28, 2017).

${ }^{41}$ See http://www.cleanroom.byu.edu/EW_ternary.phtml for bandgap value of Al0.95Ga0.05As, In0.49Ga0.51P and GaAs0.883P0.117 at $300 \mathrm{~K}$ (last accessed March 28, 2017).

${ }^{42}$ See http://www.batop.com/information/Eg_InGaAs_x_java.html for bandgap value of $\mathrm{In}_{0.145} \mathrm{Ga}_{0.855} \mathrm{As}$ at $300 \mathrm{~K}$ (last accessed March 28, 2017).

${ }^{43}$ See http://www.ioffe.ru/SVA/NSM/Semicond/ for bandgap value of GaAs at 300K (last accessed March 28, 2017).

${ }^{44}$ H. Li, P. Wolf, P. Moser, G. Larisch, A. Mutig, A. Lott, and D. H. Bimberg, "Impact of the quantum well gain-to-cavity etalon wavelength offset on the high temperature performance of high bit rate 980-nm VCSELs,” IEEE J. Quantum Electron. 50, 613-621 (2014). 